\title{
Introduction to the vol. 48, no. 2, 2021
}

\section{Maomi Ueno ${ }^{1}$}

Published online: 6 August 2021

(c) The Behaviormetric Society 2021

Welcome to the vol. 48, no. 2, 2021 of Behaviormetrika. In this issue, we have the following one invited paper, four original papers, two notes, one short note, and the special issue "e-Testing from artificial intelligence approach" by Masaki Uto and Kazuo Shigemasu.

The invited paper "Assessing Generalizability and Variability of Single-Case Design Effect Sizes using Two-Stage Multilevel Modeling including Moderators" by Mariola Moeyaert and Panpan Yang introduces an innovative meta-analytic approach, two-stage multilevel meta-analysis that considers the hierarchical structure of single-case experimental design (SCED) data (Moeyaert and Yang 2021). This approach is unique as it is suitable to include moderators at the intervention level, participant level, and study level, and is therefore especially recommended for the meta-analyst interested in moving beyond estimating the overall intervention effectiveness.

The original paper "An augmented EM algorithm for monotonic Bayesian networks using parameterized conditional probability tables" by Seyfullah Tingir and Russell Almond describes the DiBello family of models for Bayesian networks, which enforce monotonicity, and introduces an augmented EM algorithm for estimating the parameters of these models (Tingir and Almond 2021). In a calibration experiment using simulated data, the algorithm did a good job recovering the model parameters and the conditional probability tables with sample sizes as low as 400 .

The original paper "A methodology to answer to individual queries: finding relevant and robust controls" by Fernando Delbianco, Andrés Fioriti, and Fernando Tohmé presents the guidelines for an estimation and inference method aimed to answer questions to databases about new potential entries(Delbianco et al. 2021). The idea is to classify the data and find entries similar to that about which the query is posed, and use it to generate control observations so as to find the results in terms of them, instead of the entire database. This amounts to a trade-off between robustness and relevance. This allows inferring an adequate answer to a query. While a solution exists, it is not constructive and requires to explore possible alternative definitions of similarity as well as distributions that fit the selected data.

Maomi Ueno

ueno@ai.is.uec.ac.jp

1 University of Electro-Communications, Tokyo, Japan 
The original paper "Estimating the optimal treatment regime for student success programs" by Morten C. Wilke, Richard A Levine, Maureen A. Guarcello, and Juanjuan Fan challenges the cultivation of an intersection area of EDM (educational data mining) and statistics(Wilke, Levine, Guarcello, and Fan 2021). This study expands methods for estimating an optimal treatment regime (OTR) from the personalized medicine literature to educational data mining applications. As part of this development, they detail and modify the current state of the art, assess the efficacy of the approaches for student success studies, and provide practitioners the machinery to apply the methods in their specific problems. The particular interest of this study is to estimate an optimal treatment regime (OTR) for students enrolled in an introductory statistics course at San Diego State University (SDSU).

The note "A note on the three methods of item analysis" by Seock-Ho Kim, Allan S. Cohen, and Hyo Jin Eom contrasts the three methods of item analysis for multiple-choice items based on classical test theory, generalized linear modeling, and item response theory (Kim, Cohen and Eom 2021). Specifically, the methods, respectively, use a cross-classification table under classical test theory, a new baseline-category logit model under generalized linear modeling, and a multiple-choice model under item response theory. They discuss the advantages and disadvantages of each method.

The note "Estimation of odds ratio, attributable risk, relative risk, correlation coefficient and other parameters using randomized response techniques" by CheonSig Lee, Stephen A. Sedory, and Sarjinder Singh proposes two estimators of odds ratio, attributable risk, relative risk, correlation coefficient and other parameters using randomized response techniques (Lee et al. 2021). They investigate the performance of estimators based on the crossed model over those based on the simple model under the same choice of parameters.

The short note "Modeling response granularity with mixture models: a case of severity ratings in child maltreatment" by Yui Furukawa, Daiki Hojo, Jiro Sakamoto, and Kota Takaoka provides an exploratory study using mixture models with a response granularity assumption, and used the severity ratings of child maltreatment measured by slider scales with numerical feedback as a case of heaped subjective quantity (Furukawa, Hojo, Sakamoto, and Takaoka 2021). The results suggest the effectiveness of introducing mixture models to examine individual response granularity.

This issue also includes the special issue "e-Testing from artificial intelligence approach" by guest editors Masaki Uto and Kazuo Shigemasu. This special issue has three invited papers which address e-testing, a technology that uses ICT to manage and deliver tests for measuring examinee ability, using artificial intelligence technologies.

\section{References}

Delbianco F, Fioriti A, Tohmé F (2021) A methodology to answer to individual queries : finding relevant and robust controls. Behaviormetrika. https://doi.org/10.1007/s41237-021-00136-w 
Furukawa Y, Hojo D, Sakamoto J, Takaoka K (2021) Modeling response granularity with mixture models: a case of severity ratings in child maltreatment. Behaviormetrika. https://doi.org/10.1007/ s41237-021-00139-7

Kim SH, Cohen AS, Eom HJ (2021) A note on the three methods of item analysis. Behaviormetrika. https://doi.org/10.1007/s41237-021-00131-1

Lee CS, Sedory SA, Singh S (2021) Estimation of odds ratio, attributable risk, relative risk, correlation coefficient and other parameters using randomized response techniques. Behaviormetrika. https:// doi.org/10.1007/s41237-021-00135-X

Moeyaert M, Yang P (2021) Assessing generalizability and variability of single-case design effect sizes using two-stage multilevel modeling including moderators. Behaviormetrika. https://doi.org/10. 1007/s41237-021-00141-z

Tingir S, Almond R (2021) An augmented EM algorithm for monotonic Bayesian networks using parameterized conditional probability tables. Behaviormetrika. https://doi.org/10.1007/ s41237-021-00134-y

Wilke MC, Levine RA, Guarcello MA et al (2021) Estimating the optimal treatment regime for student success programs. Behaviormetrika. https://doi.org/10.1007/s41237-021-00140-0

Publisher's Note Springer Nature remains neutral with regard to jurisdictional claims in published maps and institutional affiliations. 